\title{
A Novel Method of Dexterity Analysis for a 5-DOF Manipulator
}

\author{
Wanjin Guo, ${ }^{1}$ Ruifeng Li, ${ }^{1}$ Chuqing Cao, ${ }^{2,3}$ and Yunfeng Gao ${ }^{1}$ \\ ${ }^{1}$ State Key Laboratory of Robotics and System, Harbin Institute of Technology, 92 West Dazhi Street, Nangang, Harbin 150001, China \\ ${ }^{2}$ School of Mechanical Engineering, Nanjing University of Science and Technology, Xiaolingwei 200, Nanjing 210094, China \\ ${ }^{3}$ Wuhu HIT Robot Technology Research Institute Co., Ltd., Electronic Industrial Park, Block E, JiuJiang District, Wuhu 241007, China
}

Correspondence should be addressed to Wanjin Guo; guowanjin0102@gmail.com

Received 4 October 2015; Revised 14 December 2015; Accepted 28 December 2015

Academic Editor: Yuan F. Zheng

Copyright (C) 2016 Wanjin Guo et al. This is an open access article distributed under the Creative Commons Attribution License, which permits unrestricted use, distribution, and reproduction in any medium, provided the original work is properly cited.

\begin{abstract}
The dexterity characteristics and dexterous workspace features are applied as the considerable and key kinematics properties to evaluate the manipulator performance. In this paper, a novel method of the dexterity analysis with the character of graphic dexterity and a new concept of the spatial mapping envelope surface are proposed for a 5-degree of freedom (DOF) manipulator. Additionally, for an arbitrary selected point and a dexterous workspace, the dexterity characteristics and the dexterity conditions are drawn, respectively. Finally, the dexterous workspace of this 5-DOF manipulator is obtained and it can be utilized as the basis research of this manipulator in future such as the structure optimization and motion control.
\end{abstract}

\section{Introduction}

The most obvious consideration in designing a manipulator is to feature a set of its workspace characteristics $[1,2]$. The kinematic dexterity characteristics and reachable workspace features are applied to demonstrate the fundamental properties of a manipulator. Several studies investigating kinematic dexterity indices and measures have been carried out for manipulators. The representative issues of these performance criteria for manipulators are researched.

To examine the kinematic manipulability of manipulators, several attempts have been made. The Yoshikawa manipulability ellipsoid measure was proposed to discuss the manipulating ability of robotic mechanisms in positioning and orienting end-effectors [3]. A homogeneous manipulability measure by means of the power concept was proposed for the dexterity analysis of a manipulator [4-6]. An extension to the Yoshikawa manipulability ellipsoid measure was proposed to quantify a manipulator performance in terms of dexterity and maneuverability [7]. In [8], two dexterity indices, angularity and axiality, were used to estimate the motion sensitivity of the mobile platform of a parallel manipulator.

The dexterity measures and evaluations for manipulators have been investigated. Two dexterity measures were presented for planar manipulators by Gosselin [9], which are based on the redundant kinematic equations and the minimum number of kinematic parameters, respectively. In [10], dexterity measures were presented to analyze the dexterity of an underactuated manipulator and the fault tolerance of a redundant manipulator. Two kinematic dexterity measures were proposed by Tchón and Zadarnowska [11] to determine optimal configurations and geometries of a mobile manipulator through the endogenous configuration space approach. An approach of workspace modeling with Euler parameters to explore the dexterity evaluation was proposed by Bai et al. [12] for spherical parallel manipulators with symmetrical architecture. A general and systematic approach was presented by Liu et al. [13] to evaluate the dexterity of parallel manipulators which possess mixed rotational and translational movement capabilities. In [14], weight service sphere and weight service region were defined to evaluate the dexterity of a radial symmetrical hexapod robot. Meanwhile, the dexterity of single point in workspace and the dexterity distribution in vertical and horizontal projection plane were demonstrated. In [15], a directional manipulability transmission ratio was presented to reflect the manipulability capability along the task direction for the dexterity analysis of an omnidirectional wheeled mobile manipulator.

For optimizing the kinematics dexterity of manipulators, several surveys have been published. A global conditioning 
index (GCI) was proposed for the kinematic optimization of robotic manipulators [16]. A mathematical theory was developed to optimize the kinematic dexterity of robotic mechanisms [17]. In [18], the local/global performance indexes of dexterity and manipulability are mathematically modeled and mapped to investigate the comprehensive features for the multiobjective optimization of a hybrid robotic machine tool.

Although the above studies investigating the dexterity indexes or measures are applied commonly to evaluate quantitatively the kinematic dexterity characteristics, they are not able to obviously demonstrate the topological considerations between the manipulation workspace and the performance evaluation. These studies focus on numerical or algebraic methods to characterize the relationship between the manipulator and the performance evaluation. This relationship, which cannot be revealed intuitively and obviously in the practical application, is viewed as one of the main obstacles that these numerical or algebraic methods face in attempting to feature the dexterity characteristics. More-general design principles or insights are difficult to come by using these approaches.

In this paper, the forward kinematics and the reachable workspace of a 5-DOF manipulator are derived by the Denavit and Hartenberg convention. Based on the mapping and envelope theories, a novel method with the character of graphic dexterity for dexterity analysis is proposed to evaluate this manipulator's kinematic performance. Additionally, the relationship between the dexterity characteristics and influence structure parameters can be demonstrated evidently. The dexterous workspace of this manipulator can be basically derived from the results of the dexterity analysis (these results are obtained by the proposed method) and the reachable workspace.

The proposed dexterity analysis method is developed to address the dexterity analysis problem for this 5-DOF manipulator. This method can also be applied to other manipulator structures for the dexterity analysis. This proposed method is a topological approach with clear and definite graphic dexterity character which can clearly demonstrate the dexterity characteristics and the reachable workspace features through the topological figures of the spatial mapping envelope surface and the relationship of the mapping and the orientation.

The remainder of this paper is organized as follows. The structure of a 5-DOF manipulator and its forward kinematics model are presented in Section 2. The reachable workspace of this manipulator is described in Section 3. A novel dexterity analysis method and a new concept of the spatial mapping envelope surface are proposed for the dexterity analysis of this manipulator in Section 4. The reachable workspace and the dexterous workspace of this manipulator are demonstrated in Section 5. Finally, conclusions are drawn in Section 6.

\section{Structure Description and the Forward Kinematics Model}

The structure and drive system of this 5-DOF manipulator are described. Then the forward kinematics of this manipulator is modeled based on the Denavit-Hartenberg method.
2.1. Structure Description of the Manipulator. The structure of this 5-DOF manipulator is shown in Figure 1. It is a 3T2R hybrid manipulator, which is composed of a 3-DOF (3T) parallel module and a $2-\mathrm{DOF}(2 \mathrm{R})$ serial module. The $3 \mathrm{~T}$ parallel module is constituted by the axes $J_{1}, J_{2}$, and $J_{3}$ (consisting of ball screws), which are driven by motor $M_{1}$, $M_{2}$, and $M_{3}$, respectively. The $2 \mathrm{R}$ serial module is formed by the rotational axes $J_{4}$ and $J_{5}$, which are driven by motors $M_{4}$ and $M_{5}$, respectively. The axis $J_{6}$ is taken as the endeffector which is a high-speed motorized spindle. The lengths of the $\operatorname{rod} i$ and the end-effector are denoted by $L_{i}$ and $L_{t}$, respectively. The distance between $J_{6}$ and $J_{4}$ in the horizontal direction is denoted as $e$. The nuts on the screws $S_{1}$ and $S_{2}$ are fixedly connected to the slider assemblies $A$ and $C$, respectively. The slider assembly $B$ is rotated through a rotation shaft which is connected to $A$, and the slider assembly $D$ is rotated in the same way. The pairs of slider assemblies $B$ and $D$ are moved along the slide plate which goes across $S_{1}$ and $S_{2}$. The turntable, which can rotate within a certain range, is fixedly connected to the slide plate. In the meantime, the turntable is moved along the symmetry axis of $S_{1}$ and $S_{2}$ in the horizontal direction.

It is worth noting that the first two DOFs of this manipulator (i.e., two translational movements in the horizontal plane) are achieved by the parallel mechanism which is a coupled mechanism. It is driven in parallel by $M_{1}$ and $M_{2}$ as shown in Figure 1. This coupled mechanism achieves simultaneously two translational movements in the horizontal plane by two small power motors. Furthermore, the orientation and the position of the end-effector are regulated by two rotation joints and three ball-screw drives, respectively. More details of the mobility analysis and the structure analysis for this 5DOF manipulator are described in our paper [19].

2.2. Modeling of the Forward Kinematics. The D-H reference frame [20] is established as shown in Figure 2. The rotation angles of $J_{1}, J_{2}, J_{3}, J_{4}$, and $J_{5}$ are represented by $\varphi_{1}, \varphi_{2}, \varphi_{3}$, $\varphi_{4}$, and $\varphi_{5}$, respectively. The strokes of the nut along $S_{1}, S_{2}$, and $S_{3}$ are represented by $X_{1}, X_{2}$, and $X_{3}$, respectively. The rotation angle of the axis $J_{t}$ and link 1 is denoted as $\alpha . L_{01}$ represents the distance between the point $\mathrm{O}_{2}$ and the point $\mathrm{O}_{1}$ in the vertical direction when the nut is located at the top starting point along $S_{3}$. These variables are related by

$$
\begin{aligned}
\alpha & =\arctan \left(\frac{\left(X_{2}-X_{1}\right)}{L_{1}}\right), \\
X_{1} & =\frac{s_{x} \varphi_{1}}{(2 \pi)}, \\
X_{2} & =\frac{s_{x} \varphi_{2}}{(2 \pi)}, \\
X_{3} & =\frac{s_{x} \varphi_{3}}{(2 \pi)},
\end{aligned}
$$

where the constant $s_{x}$ represents the pitch of $S_{1}$ and $S_{2}$ and the constant $s_{z}$ represents the pitch of $S_{3}$.

Link 1 is driven in parallel by $M_{1}$ and $M_{2}$ as shown in Figures 1 and 2. This link is moved along the axis $X_{1}$ which 


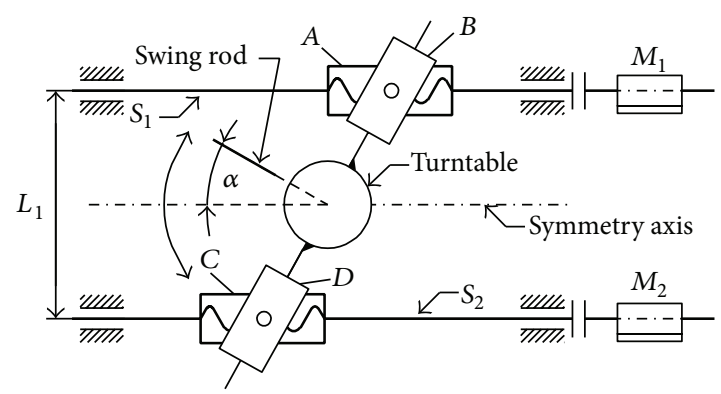

(a) Top view

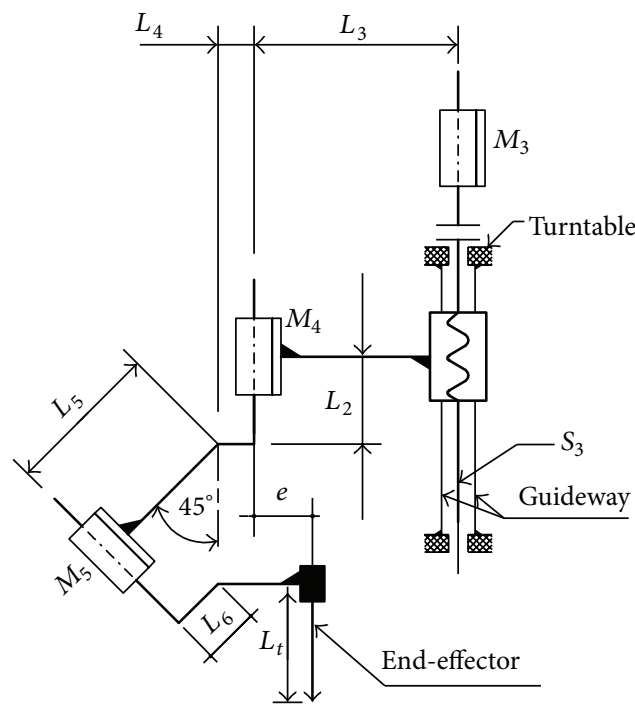

(b) Front view

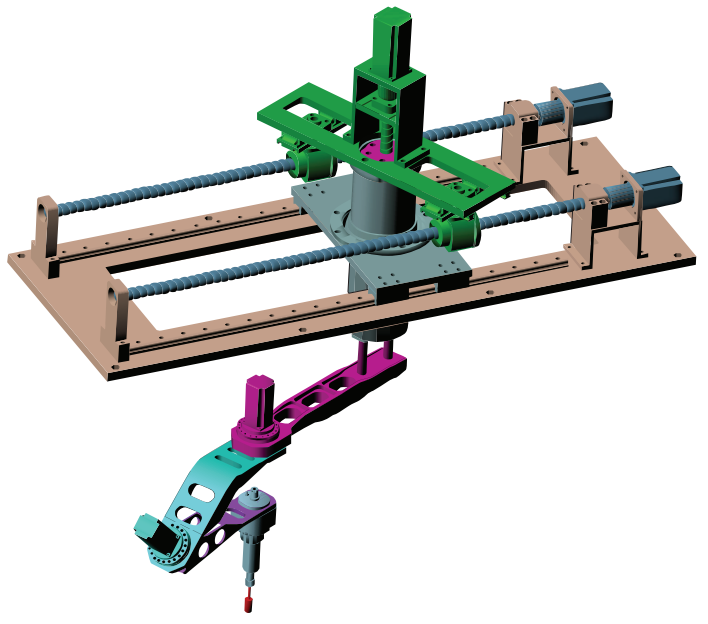

(c) 3D model

FIGURE 1: Manipulator structure.

is also the symmetry axis of $S_{1}$ and $S_{2}$. It is also rotated about $J_{t}$ with the turntable when the strokes of $A$ and $C$ along the screw are different. Hence, a coupled movement is generated. The movement direction of link 1 along the axis $X_{1}$ is always paralleled to $X_{0}$ while this link is rotated about $Z_{1}$ and $J_{t}$. According to this movement relationship, the homogeneous transformation matrix ${ }^{0} T_{1}$ which relates the spatial displacement of the reference frame 1 to the base reference frame 0 is directly given as

$$
{ }^{0} T_{1}=\left[\begin{array}{cccc}
\cos \alpha & -\sin \alpha & 0 & \frac{\left(X_{1}+X_{2}\right)}{2} \\
\sin \alpha & \cos \alpha & 0 & 0 \\
0 & 0 & 1 & 0 \\
0 & 0 & 0 & 1
\end{array}\right] .
$$

The geometric parameters of this manipulator for links 2 , 3,4 , and 5 (tool) shown in Figures 1 and 2 are listed in Table 1.
The homogeneous transformation matrix ${ }^{i-1} T_{1}$ is conducted to provide a representation of reference frame $i$ relative to reference frame $i-1$ according to the $\mathrm{D}-\mathrm{H}$ reference frames and the geometric parameters. It is given as

$$
\begin{aligned}
& { }^{i-1} T_{1} \\
& =\left[\begin{array}{cccc}
\cos \theta_{i} & -\cos \alpha_{i} \cdot \sin \theta_{i} & \sin \alpha_{i} \cdot \sin \theta_{i} & a_{i} \cdot \cos \theta_{i} \\
\sin \theta_{i} & \cos \alpha_{i} \cdot \cos \theta_{i} & -\sin \alpha_{i} \cdot \cos \theta_{i} & a_{i} \cdot \sin \theta_{i} \\
0 & \sin \alpha_{i} & \cos \alpha_{i} & d_{i} \\
0 & 0 & 0 & 1
\end{array}\right],
\end{aligned}
$$

where $i=2, \ldots, 5, i \in N$.

By successive multiplications of the transformation matrices, the equivalent homogeneous transformation matrix ${ }^{0} T_{5}$ which relates the spatial displacement of the tool frame to the base reference frame is obtained as

$$
{ }^{0} T_{5}={ }^{0} T_{1}{ }^{1} T_{2}{ }^{2} T_{3}{ }^{3} T_{4}{ }^{4} T_{5} .
$$


TABLE 1: Geometric parameters of links.

\begin{tabular}{lcccc}
\hline Link $i$ & $\theta_{i}$ & $\alpha_{i}$ & $d_{i}$ & $a_{i}$ \\
\hline 2 & 0 & 0 & $X_{3}+L_{01}$ & $L_{3}$ \\
3 & $-\pi / 2+\varphi_{4}$ & $\pi / 4$ & $L_{2}+L_{4}+\sqrt{2} L_{5}$ & 0 \\
4 & $\pi+\varphi_{5}$ & $\pi / 4$ & $\sqrt{2} e$ & 0 \\
5 & 0 & 0 & 0 & 0 \\
\hline
\end{tabular}

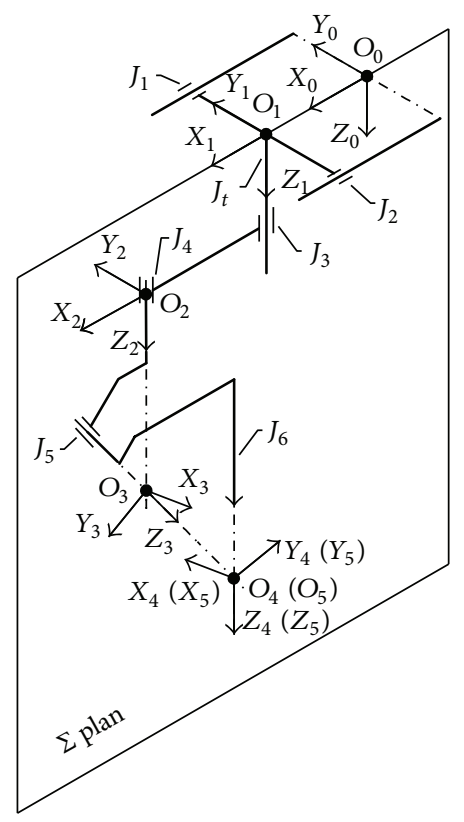

Figure 2: Denavit-Hartenberg reference frame.

\section{Representation of the Reachable Workspace}

More generally, the workspace of a manipulator can be represented as a total volume swept out by the end-effector as the manipulator executes all possible motions [21]. The workspace is determined by the geometry of the manipulator and the limits of the joint motions. In order to analyze the dexterity characteristics and the dexterous workspace in the following sections, the reachable workspace corresponding to the operation point of the end-effector is derived by the following steps.

Suppose that a position vector $\vec{P}=\left[x_{P}, y_{P}, z_{P}\right]^{T}$ represents the operation point $P$ in the tool reference frame. According to the position of $P$ in the tool reference frame, $x_{P}=0, y_{P}=0$, and $z_{P}=L_{P}$ are obtained, where $L_{P}$ defined as the tool compensation which is the value of the tool operating point in $Z_{5}$ axis is given as

$$
L_{P}=L_{t}-\left(L_{4}+\sqrt{2} L_{6}+e\right) .
$$

The reachable workspace of the operation point $P$ is obtained based on the forward kinematics model. By successive multiplication of the transformation matrices $\left[x_{0}, y_{0}, z_{0}, 1\right]^{T}={ }^{0} T_{1} \cdot{ }^{1} T_{2} \cdot{ }^{2} T_{3} \cdot{ }^{3} T_{4} \cdot{ }^{4} T_{5} \cdot\left[x_{P}, y_{P}, z_{P}, 1\right]^{T}$, the expression of this reachable workspace is derived. In the base reference frame, a space manifold $\Sigma_{\mathrm{O}_{0} P}$ is obtained as

$$
\Sigma_{\mathrm{O}_{0} P}:\left\{\begin{array}{l}
x_{0}=\frac{X_{1}+X_{2}}{2}-\frac{\sqrt{2}}{2} L_{P} \sin \left(\alpha+\varphi_{4}\right) \sin \varphi_{5}-\cos \left(\alpha+\varphi_{4}\right)\left(\frac{1-\cos \varphi_{5}}{2} L_{P}+e\right)+L_{3} \cos \alpha \\
y_{0}=\frac{\sqrt{2}}{2} L_{P} \cos \left(\alpha+\varphi_{4}\right) \sin \varphi_{5}-\sin \left(\alpha+\varphi_{4}\right)\left(\frac{1-\cos \varphi_{5}}{2} L_{P}+e\right)+L_{3} \sin \alpha \\
z_{0}=X_{3}+L_{01}+\left(L_{2}+L_{4}+\sqrt{2} L_{5}\right)+e+\frac{1+\cos \varphi_{5}}{2} L_{P} .
\end{array}\right.
$$

\section{The Proposed Method for Dexterity Analysis}

In this section, based on the mapping and envelope theory, a novel dexterity analysis method with the character of graphic dexterity and a new concept of the spatial mapping envelope surface are proposed for the dexterity analysis of this manipulator. The proposed dexterity analysis method can be also applied to other manipulator structures to address the dexterity analysis problem. The brief outline of the proposed method is stated as follows.

The reachable workspace of the reference point is partitioned into a regional workspace volume and an orientation workspace volume based on the effect of the orientation; that is, the space manifold of the reachable workspace is decomposed into a regional portion and an orientation portion. Then, an arbitrary point is selected in this reachable workspace and this point is mapped to a spatial mapping envelope surface with a matching relationship. By analyzing the mapping envelope features and the geometry meanings of this spatial mapping envelope surface, the dexterity characteristics of this arbitrary selected point are drawn. Finally, the condition that the arbitrary selected point is a strict dexterity point with the strict dexterity performance is deduced. Meanwhile, the condition that a dexterous workspace is a strict dexterous workspace with the strict dexterity performance is also derived.

4.1. Decomposition of the Reachable Workspace. The workspace can be defined by an envelope which is essentially of one or two types, either a manifold or a surface that is smooth almost everywhere, that is, smooth everywhere except for a set of points of measure zero in the Lebesgue sense [22]. In this paper, based on the influence factors for reachable 
workspace, the space manifold $\Sigma_{\mathrm{O}_{0} P}$ corresponding to the reachable workspace of $P$ is decomposed into a regional portion $\Sigma_{\mathrm{O}_{0} P}^{r}$ and an orientation portion $\Sigma_{\mathrm{O}_{0} P}^{o}$, and it is given as

$$
\Sigma_{\mathrm{O}_{0} P}=\Sigma_{\mathrm{O}_{0} P}^{r}+\Sigma_{\mathrm{O}_{0} P}^{o},
$$

where

$$
\begin{aligned}
& \Sigma_{\mathrm{O}_{0} P}^{r}:\left\{\begin{array}{l}
x_{0-T}=\frac{X_{1}+X_{2}}{2}+L_{3} \cos \alpha \\
y_{0-T}=L_{3} \sin \alpha \\
z_{0-T}=X_{3}+L_{01}+\left(L_{2}+L_{4}+\sqrt{2} L_{5}\right)+e,
\end{array}\right. \\
& \Sigma_{\mathrm{O}_{0}-P}^{o}:\left\{\begin{array}{l}
x_{0-R}=-\frac{\sqrt{2}}{2} L_{P} \sin \left(\alpha+\varphi_{4}\right) \sin \varphi_{5}-\cos \left(\alpha+\varphi_{4}\right)\left(\frac{1-\cos \varphi_{5}}{2} L_{P}+e\right) \\
y_{0-R}=\frac{\sqrt{2}}{2} L_{P} \cos \left(\alpha+\varphi_{4}\right) \sin \varphi_{5}-\sin \left(\alpha+\varphi_{4}\right)\left(\frac{1-\cos \varphi_{5}}{2} L_{P}+e\right) \\
z_{0-R}=\frac{1+\cos \varphi_{5}}{2} L_{P} .
\end{array}\right.
\end{aligned}
$$

According to the expressions of the regional portion and the orientation portion, it can be found that the regional workspace volume of the reachable workspace is mostly provided by the former, and a trivial or even negligible share is provided by the latter. Meanwhile, the orientation workspace volume is entirely provided by the latter. Hence, the dexterity of an arbitrary operation point in the reachable workspace is completely determined by the latter.

The envelope surface of $\Sigma_{\mathrm{O}_{0} P}^{o}$ is obtained by eliminating the parameter variables $\alpha, \varphi_{4}$, and $\varphi_{5}$ from its expression. $r_{1}$ and $z_{0-R}$ are defined as the radius and the axis of the circular cross section for $\Sigma_{\mathrm{O}_{0} P}^{o}$, respectively. $Q_{1}$ is defined as the geometric center of the envelope surface $\Sigma_{\mathrm{O}_{0} P}^{o}$ and the center of the maximum circular cross-section. $r_{1-\max }$ and $h_{1}$ are defined as the radius of the maximum circular cross-section and the height of the envelope surface in the $z_{0-R}$ direction, respectively. The expression of the envelope circular crosssection in the $z_{0-R}$ direction is given as

$$
\begin{aligned}
& r_{1}^{2}=x_{0-R}^{2}+y_{0-R}^{2}=\left(L_{P}+e\right)^{2}-\left(z_{0-R}+e\right)^{2}+e^{2}, \\
& z_{0-R} \in\left[0,\left|L_{P}\right|\right] \quad\left(L_{P} \geq 0\right) \text { or } z_{0-R} \in\left[-\left|L_{P}\right|, 0\right] \quad\left(L_{P}<0\right),
\end{aligned}
$$

where $\left|L_{P}\right|$ represents the absolute value of the parameter $L_{P}$ and the value of parameter $e$ is nonnegative.

To illustrate the envelope surface shape of $\Sigma_{\mathrm{O}_{0} P}^{o}$, an example of $L_{P}=-70 \mathrm{~mm}$ and $e=30 \mathrm{~mm}$ is given. $r_{1-\max }=$ $50 \mathrm{~mm}, h_{1}=70 \mathrm{~mm}$, and $Q_{1}=[0,0,-30]^{T}$ are derived. The shape of this envelope surface is shown in Figure 3.

4.2. Dexterity Analysis of an Operation Point P. Several conventions for the dexterity properties are made. A reference operation point $P_{1}$ within the reachable workspace is called a strict dexterity point with the strict dexterity when the endeffector can reach $P_{1}$ in any arbitrary orientation. Likewise, $P_{2}$ is called a finite dexterity point with the finite dexterity when the end-effector can only reach $P_{2}$ in a certain range of orientation. A workspace is called a strictly dexterous workspace if any arbitrary point within this workspace is the strict dexterity point.

In order to analyze the kinematic dexterity characteristics of an operation point and the dexterity features of the reachable workspace, an arbitrary operation point $P$ is selected on the envelope surface of the reachable workspace. According to the envelope surface and the shape of $\Sigma_{\mathrm{O}_{0}-}^{o}$, the center of the circular cross section at $z_{0-R}=0$ coincides with the origin $\mathrm{O}_{5}$ of the tool reference frame. The distance between an arbitrary operation point $P$ on $\Sigma_{O_{0}-P}^{o}$ and $O_{5}$ is written as

$$
\begin{aligned}
& R=\sqrt{x_{0-R}^{2}+y_{0-R}^{2}+z_{0-R}^{2}}=\sqrt{\left(L_{P}+e\right)^{2}-2 e z_{0-R}}, \\
& z_{0-R} \in\left[0,\left|L_{P}\right|\right] \quad\left(L_{P} \geq 0\right) \text { or } z_{0-R} \in\left[-\left|L_{P}\right|, 0\right] \quad\left(L_{P}<0\right) .
\end{aligned}
$$

Based on the concepts of mapping and envelope, a novel spatial mapping envelope surface is established. An arbitrary operation point $P$ is selected on the envelope surface $\Sigma_{\mathrm{O}_{0} P}^{o}$ of the reachable workspace. Then, rotating the end-effector at the selected point $P$ in any arbitrarily reachable orientation, a novel spatial mapping envelope surface $\Sigma_{\mathrm{O}_{5}}^{o}$ which consists of mapping points $O_{5}$ is mapped by $P$ with the distance $R=$ $\sqrt{\left(L_{P}+e\right)^{2}-2 e z_{0-R}}$.

$r_{2}$ and $z_{0-R}$ are defined as the radius and the axis of the circular cross section for the spatial mapping envelope, respectively. The circular cross section expression of the mapping envelope in the $z_{0-R}$ direction is given as

$$
\begin{aligned}
& r_{2}^{2}=x_{\mathrm{O}_{5}}^{2}+y_{\mathrm{O}_{5}}^{2}=\left(L_{P}+e\right)^{2}-\left(z_{0-R}+e\right)^{2}+e^{2}, \\
& z_{0-R} \in\left[0,\left|L_{P}\right|\right]\left(L_{P} \geq 0\right) \text { or } z_{0-R} \in\left[-\left|L_{P}\right|, 0\right] \quad\left(L_{P}<0\right) .
\end{aligned}
$$

To demonstrate the shape of a mapping envelop surface $\Sigma_{\mathrm{O}_{5}}^{o}$ and the relationship between the mapping and the orientation, two examples are provided. 

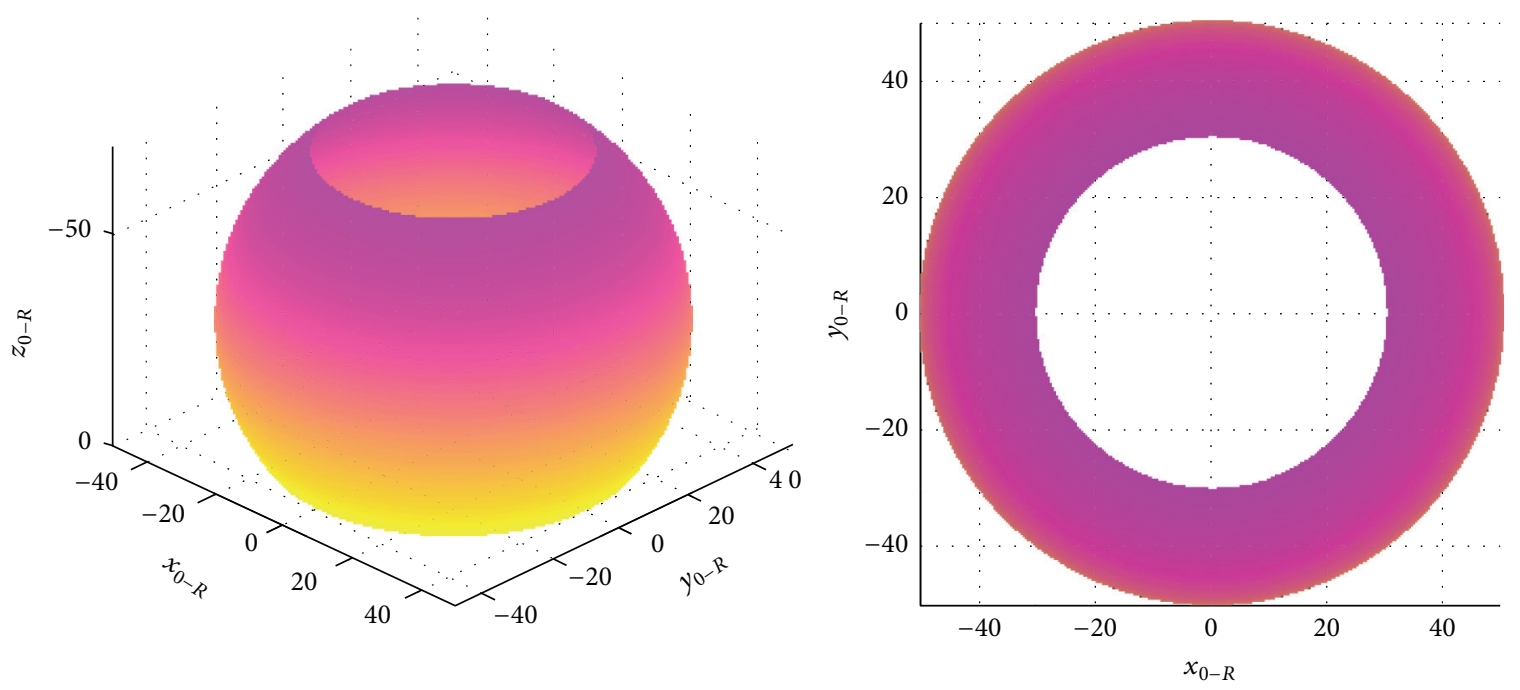

FIGURE 3: Shape of envelope surface.

Example $1\left(L_{P}=-70 \mathrm{~mm}, e=30 \mathrm{~mm}\right)$. According to the mapping relationship, a spatial mapping envelope surface is obtained, as shown in Figure 4(a). There exists a cavity region on $\Sigma_{\mathrm{O}_{5}}^{o}$. An arbitrary mapping point $\mathrm{O}_{5}$ which is located on $\Sigma_{\mathrm{O}_{5}}^{o}$ can be mapped by $P$ in an orientation. However, an arbitrary mapping point $\mathrm{O}_{5}$ which is situated in the cavity region does not have a counterpart on $\Sigma_{\mathrm{O}_{5}}^{o}$; that is, the corresponding orientations of the end-effector are unreachable. Hence, the condition of $P$ with dexterity properties in a certain orientation is that the mapping point $\mathrm{O}_{5}$ has a counterpart on $\Sigma_{\mathrm{O}_{5}}^{o}$ in this orientation. The relationship between the mapping and the orientation is shown in Figure 4(b).

Example $2\left(L_{P}=-70 \mathrm{~mm}, e=0 \mathrm{~mm}\right)$. Similarly, another spatial mapping envelope surface is given, as shown in Figure 5(a). It is an entire and continuous hemisphere without a cavity region. An arbitrary mapping point $O_{5}$ which is located on $\Sigma_{\mathrm{O}_{5}}^{o}$ can be mapped by $P$ in an orientation; that is, the corresponding orientations of the end-effector are reachable. The relationship between the mapping and the orientation is shown in Figure 5(b).

Through the proposed method, by analyzing the mapping envelope features and mapping figures, the relationship between the manipulator and the performance evaluation and the relationship between the mapping and the orientation, with the character of graphical dexterity, are obviously demonstrated, respectively. Based on the proposed dexterity analysis method, the conditions that the arbitrary selected point is a strict dexterity point with the strict dexterity performance and that a dexterous workspace is a strict dexterous workspace with the strict dexterity performance are also derived, respectively.

(i) When a cavity region exists on $\Sigma_{\mathrm{O}_{5}}^{o}$, some corresponding orientations of the end-effector are unreachable; $P$ can only be reached by the end-effector within a certain range in an arbitrary orientation; that is, $P$ is a finite dexterity point with finite dexterity.
TABLE 2: The assumed manipulator structure parameters (mm).

\begin{tabular}{cccccccccc}
\hline$L_{1}$ & $L_{2}$ & $L_{3}$ & $L_{4}$ & $L_{5}$ & $L_{6}$ & $e$ & $L_{t}$ & $L_{01}$ & $s_{x} / s_{z}$ \\
\hline 420 & 50 & 450 & 180 & 180 & 80 & 0 & 225 & 400 & 10 \\
\hline
\end{tabular}

However, when the entire and continuous hemisphere $\Sigma_{\mathrm{O}_{5}}^{o}$ without a cavity region is mapped, all corresponding orientations of the end-effector are reachable; $P$ can be reached by the end-effector in any arbitrary orientation; that is, this is the necessary and sufficient condition that $P$ is a strict dexterity point with the strict dexterity.

(ii) A strictly dexterous workspace exists if and only if an entire and continuous hemisphere $\Sigma_{\mathrm{O}_{5}}^{o}$ without a cavity region can be mapped by any arbitrary point in this workspace; that is, any arbitrary point in this workspace is a strict dexterity point.

\section{Workspace Analysis}

The manipulator workspace is classified into two components, that is, the reachable workspace and the dexterous workspace [23]. In this section, the reachable workspace corresponding to the selected point $P$ for this 5-DOF manipulator is obtained; then the dexterous graphic workspace can be derived on the basis of the results of the dexterity analysis (these results are obtained by the proposed method) and the reachable workspace.

5.1. Depiction of the Reachable Workspace. The reachable workspace of this manipulator is obtained through MATLAB simulation based on the forward kinematics solution with the assumed structure parameters (Table 2). The reachable workspace of the selected point $P$ is shown in Figure 6. The top view and the front view of Figure 6 show the range in 

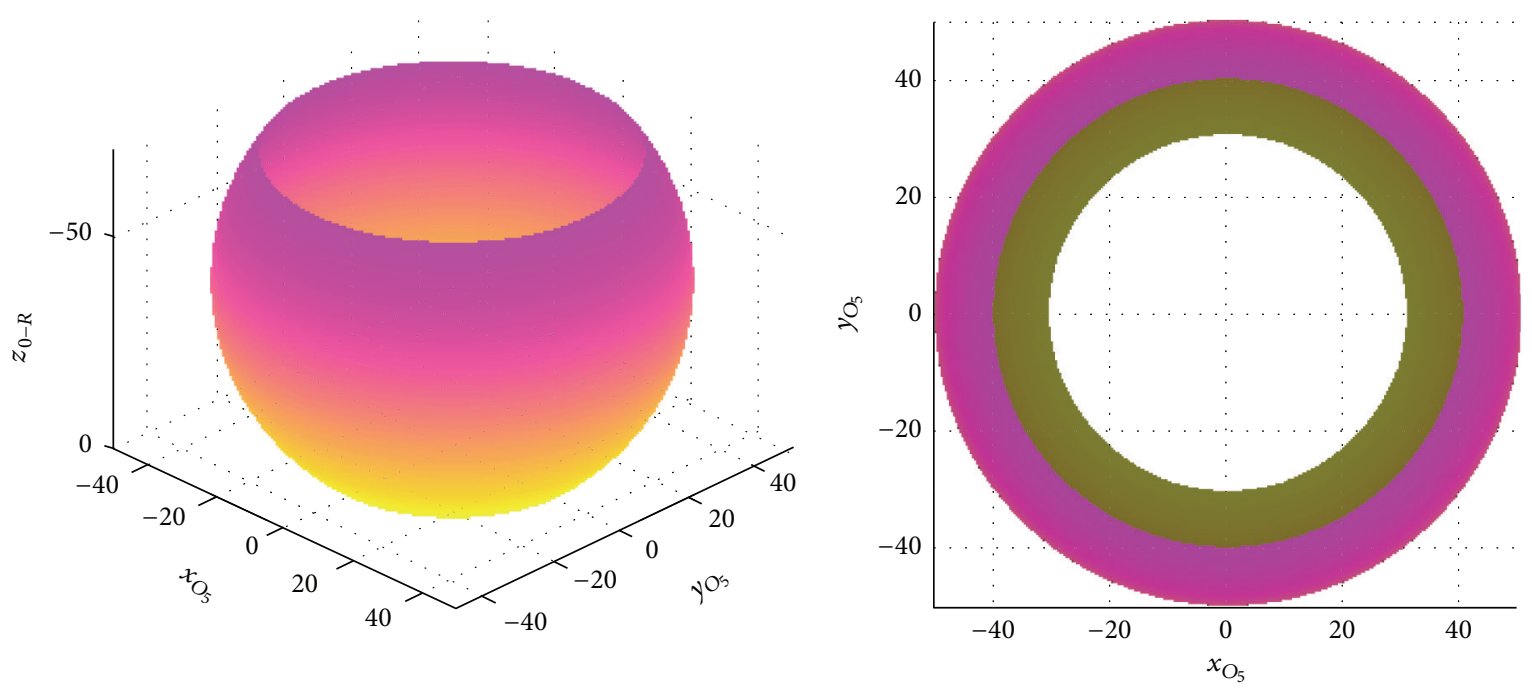

(a) Mapping envelope

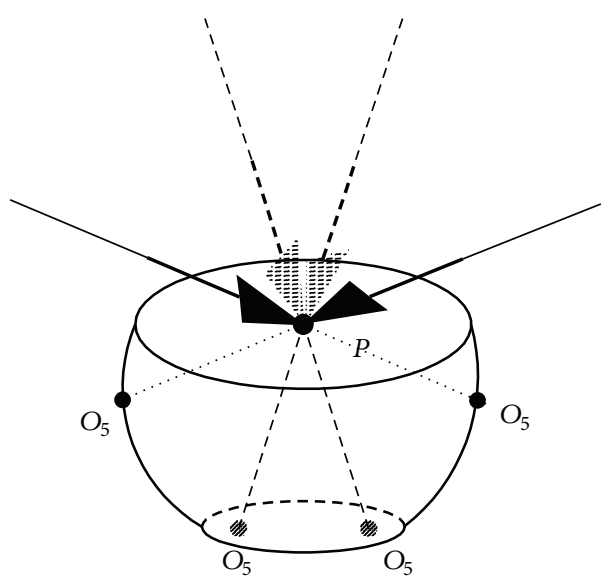

(b) Mapping relationship

Figure 4: The example of $L_{P}=-70$ and $e=30$.

the $X, Y$, and $Z$ direction of this manipulator reachable workspace.

Note that the shapes of the left end and the right end (in the top view of Figure 6) are different. This is caused by the sway of the swing rod. The sway values are $L_{3} \cos \alpha$ and $L_{3} \sin \alpha$ in the $X$ and $Y$ directions, respectively. In the start phase, $\alpha$ increases from zero to the maximum attainable value, and the sway values in the $X$ and $Y$ directions gradually decrease and increase, respectively. In the end phase, $\alpha$ decreases from the maximum attainable value to zero, and the sway values in the $X$ and $Y$ directions gradually increase and decrease, respectively. The mutual variation relationship is shown in Figure 6(a).

5.2. Determination of the Dexterous Workspace. When a strictly dexterous workspace exists, the dexterous workspace is derived on the basis of the above-obtained reachable workspace and the results of the dexterity analysis. The process is carried out as the following steps.
The boundary of the reachable workspace of $P$ is contracted towards the inner area with two times of $r_{1-\max }$ in the top view, as shown in Figure 7(a). Then, in the front view, the boundaries of the left and right edges and the boundaries of the upper and lower edges are contracted towards the inner area with two times of $r_{1-\max }$ and two times of $h_{1}$, respectively, as shown in Figure 7(b).

\section{Conclusions}

In this paper, a dexterity analysis method is proposed to address the dexterity analysis and the dexterity characteristics for a 5-DOF manipulator, through a geometric and topological approach with the character of graphic dexterity. Based on the proposed method, the relationship of the manipulator and the performance evaluation and the relationship of the mapping and the orientation are demonstrated obviously with the character of graphical dexterity, respectively. To summarize, the major contributions of this paper are as follows. 

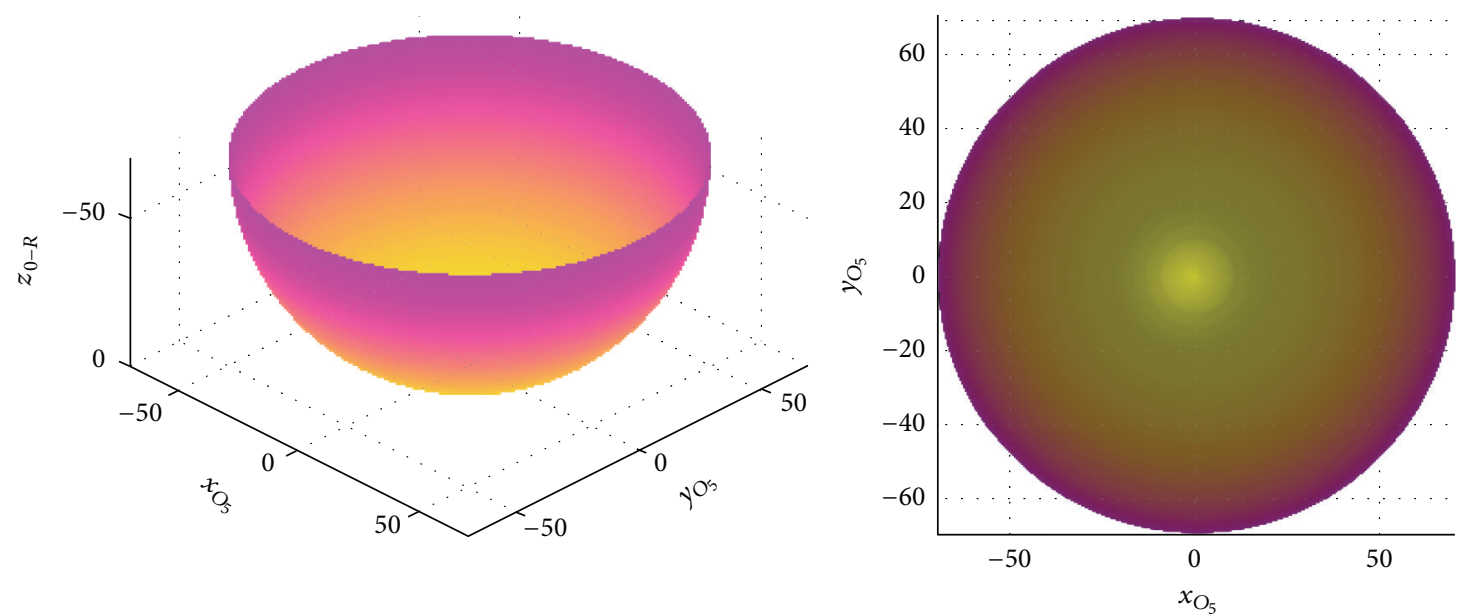

(a) Mapping envelope

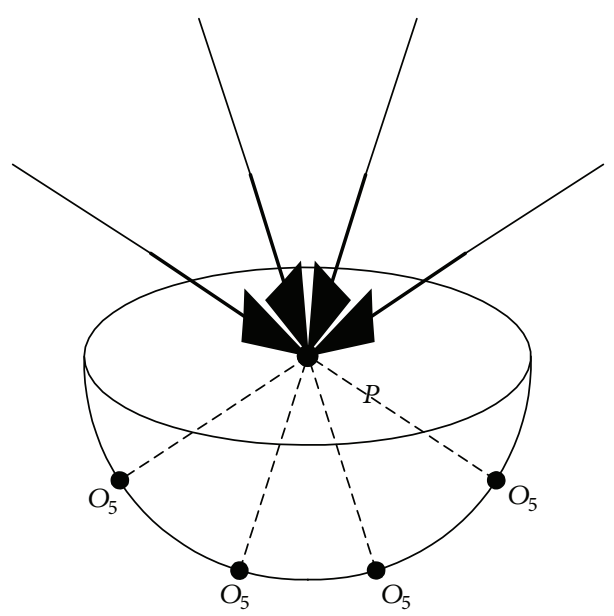

(b) Mapping relationship

FIgURE 5: The example of $L_{P}=-70$ and $e=0$.

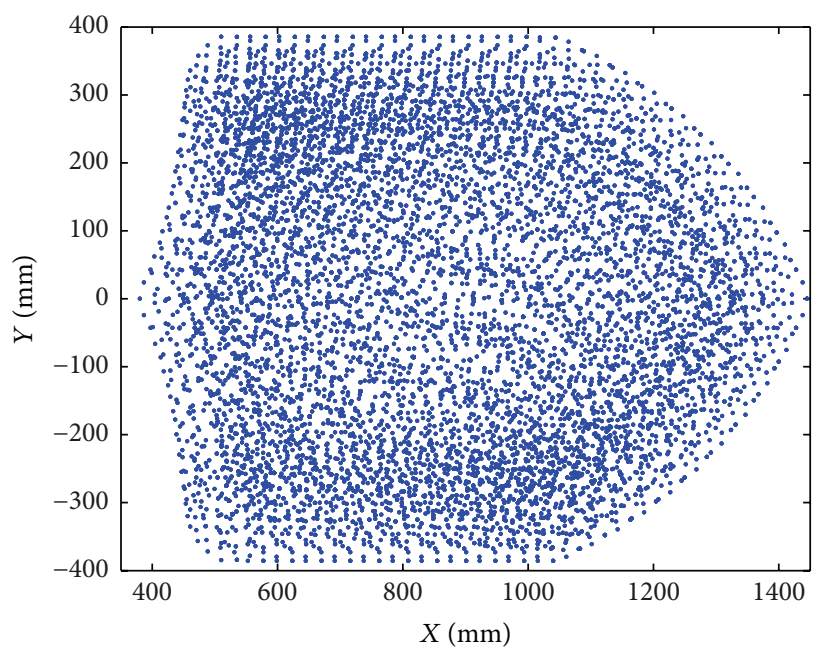

(a) Top view

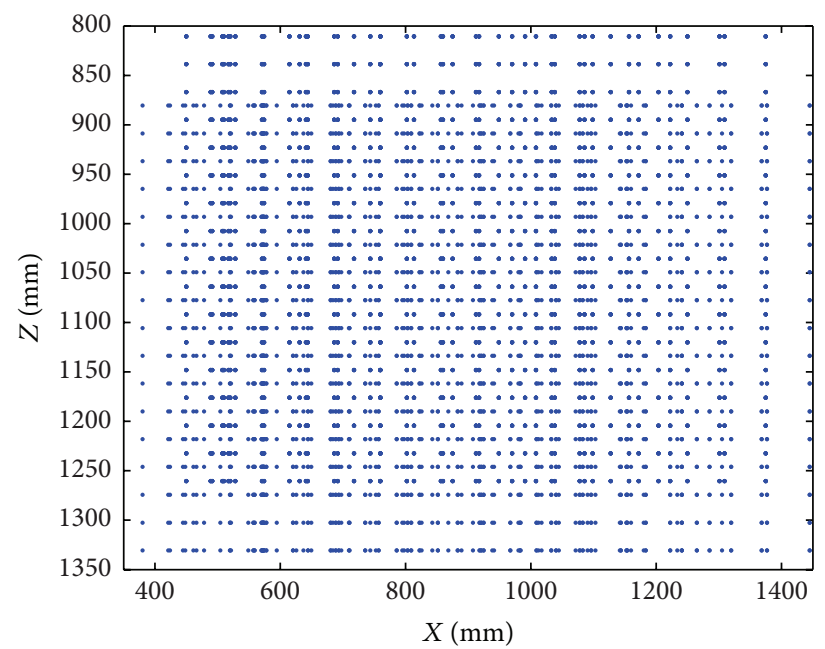

(b) Front view

Figure 6: Reachable workspace. 


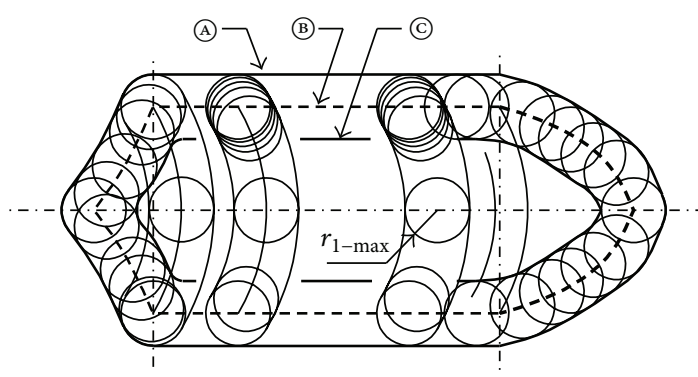

(a) Top view

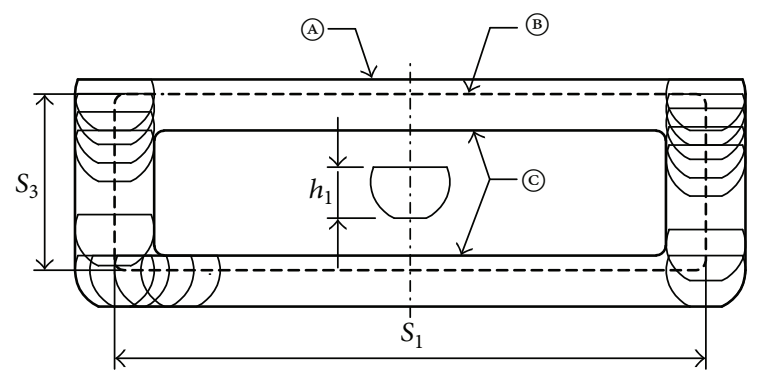

(b) Front view

Figure 7: Projection of dexterous workspace of $P$. The symbols (A), (B), and (C) represent the boundary of the reachable workspace of $P$, the locus of the geometric center $Q_{1}$, and the boundary of the dexterous workspace of the selected point $P$, respectively.

(1) The structure of a 5-DOF manipulator and its forward kinematics model are derived. The reachable workspace of this manipulator is presented for the dexterity analysis and dexterous workspace determination.

(2) A novel method of the dexterity analysis with the character of graphic dexterity and a new concept of the spatial mapping envelope surface are proposed for this 5-DOF manipulator. The proposed method can be also applied to other manipulator structures for the dexterity analysis problem. The dexterity characteristics of an arbitrary selected point are given. Meanwhile, the conditions that the arbitrary selected point is a strict dexterity point with the strict dexterity performance and that a dexterous workspace is a strict dexterous workspace with the strict dexterity performance are derived, respectively.

(3) The dexterous graphic workspace of this 5-DOF manipulator is determined. It might serve as the base research of this manipulator for the structure optimization and motion control.

In future work, the structure optimization, dynamics problem, and motion control will be further considered.

\section{Conflict of Interests}

The authors declare that there is no conflict of interests regarding the publication of this paper.

\section{Acknowledgment}

This work was supported by the National High-Tech R\&D Program (863 Program) of China (no. 2013AA040902-2).

\section{References}

[1] D. Lau, J. Eden, D. Oetomo, and S. K. Halgamuge, "Musculoskeletal static workspace analysis of the human shoulder as a cable-driven robot," IEEE/ASME Transactions on Mechatronics, vol. 20, no. 2, pp. 978-984, 2015.
[2] S. Seriani, M. Seriani, and P. Gallina, "Workspace optimization for a planar cable-suspended direct-driven robot," Robotics and Computer-Integrated Manufacturing, vol. 34, pp. 1-7, 2015.

[3] T. Yoshikawa, "Manipulability of robotic mechanisms," The International Journal of Robotics Research, vol. 4, no. 2, pp. 39, 1985.

[4] I. Mansouri and M. Ouali, "A new homogeneous manipulability measure of robot manipulators, based on power concept," Mechatronics, vol. 19, no. 6, pp. 927-944, 2009.

[5] I. Mansouri and M. Ouali, "The power manipulability-a new homogeneous performance index of robot manipulators," Robotics and Computer-Integrated Manufacturing, vol. 27, no. 2, pp. 434-449, 2011.

[6] I. Mansouri and M. Ouali, "Quaternion representation of the power manipulability," Transactions of the Canadian Society for Mechanical Engineering, vol. 35, no. 2, pp. 309-336, 2011.

[7] N. Vahrenkamp and T. Asfour, "Representing the robot's workspace through constrained manipulability analysis," Autonomous Robots, vol. 38, no. 1, pp. 17-30, 2015.

[8] J. J. Cervantes-Sánchez, J. M. Rico-Martínez, and V. H. PérezMuñoz, "Two natural dexterity indices for parallel manipulators: angularity and axiality," Journal of Mechanisms and Robotics, vol. 6, no. 4, Article ID 041007, 13 pages, 2014.

[9] C. M. Gosselin, "Dexterity indices for planar and spatial robotic manipulators," in Proceedings of the IEEE International Conference on Robotics and Automation, vol. 1, pp. 650-655, IEEE, Cincinnati, Ohio, USA, May 1990.

[10] R. G. Roberts, "The dexterity and singularities of an underactuated robot," Journal of Robotic Systems, vol. 18, no. 4, pp. 159-169, 2001.

[11] K. Tchón and K. Zadarnowska, "Kinematic dexterity of mobile manipulators: an endogenous configuration space approach," Robotica, vol. 21, no. 5, pp. 521-530, 2003.

[12] S. Bai, M. R. Hansen, and T. O. Andersen, "Modelling of a special class of spherical parallel manipulators with Euler parameters," Robotica, vol. 27, no. 2, pp. 161-170, 2009.

[13] H. Liu, T. Huang, and D. G. Chetwynd, "A method to formulate a dimensionally homogeneous jacobian of parallel manipulators," IEEE Transactions on Robotics, vol. 27, no. 1, pp. 150-156, 2011.

[14] K. Xu and X. Ding, "Gait analysis of a radial symmetrical hexapod robot based on parallel mechanisms," Chinese Journal of Mechanical Engineering, vol. 27, no. 5, pp. 867-879, 2014.

[15] B. Du, J. Zhao, and C. Song, "Dexterity analysis for omnidirectional wheeled mobile manipulator based on double 
quaternion," Chinese Journal of Mechanical Engineering (English Edition), vol. 26, no. 3, pp. 585-593, 2013.

[16] C. Gosselin and J. Angeles, "A global performance index for the kinematic optimization of robotic manipulators," Journal of Mechanical Design, vol. 113, no. 3, pp. 220-226, 1991.

[17] F. C. Park and R. W. Brockett, "Kinematic dexterity of robotic mechanisms," The International Journal of Robotics Research, vol. 13, no. 1, pp. 1-15, 1994.

[18] Z. Gao and D. Zhang, "Performance analysis, mapping, and multiobjective optimization of a hybrid robotic machine tool," IEEE Transactions on Industrial Electronics, vol. 62, no. 1, pp. 423-433, 2015.

[19] W. J. Guo, R. F. Li, C. Q. Cao, and Y. F. Gao, "Kinematics analysis of a novel 5-DOF hybrid manipulator," International Journal of Automation Technology, vol. 9, no. 6, pp. 765-774, 2015.

[20] J. Denavit, "A kinematic notation for lower-pair mechanisms based on matrices," Transactions of the ASME. Journal of Applied Mechanics, vol. 22, pp. 215-221, 1955.

[21] B. Siciliano and O. Khatib, Springer Handbook of Robotics, Springer, Berlin, Germany, 2008.

[22] K. Hoffman, Analysis in Euclidean Space, Courier Dover Publications, Mineola, NY, USA, 2013.

[23] A. Kumar and K. J. Waldron, "The workspaces of a mechanical manipulator," Journal of Mechanical Design, vol. 103, no. 3, pp. 665-672, 1981. 


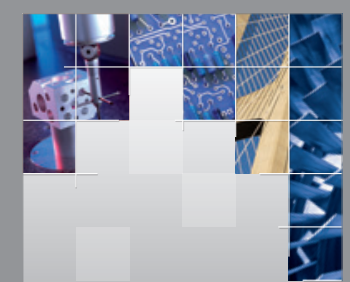

\section{Enfincering}
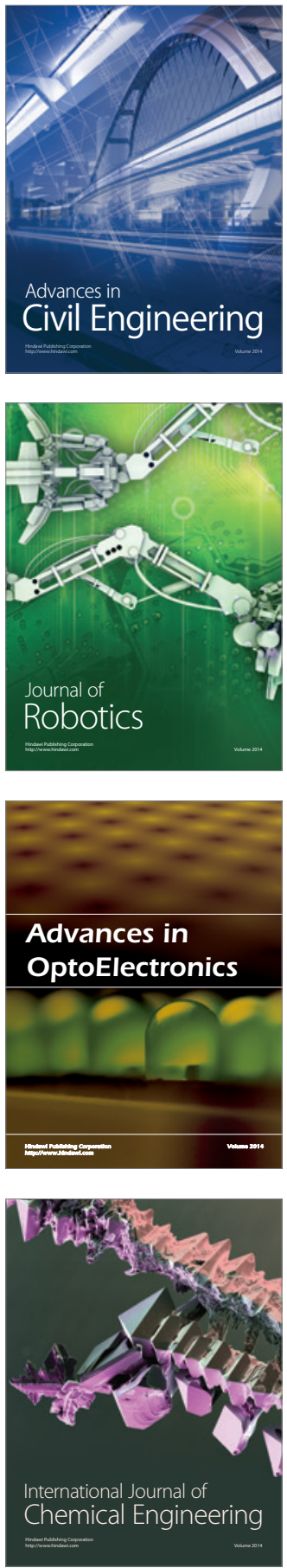

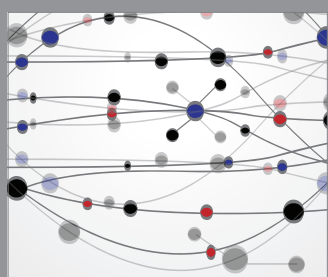

The Scientific World Journal

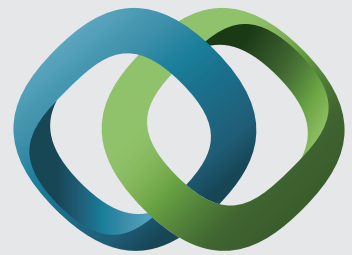

\section{Hindawi}

Submit your manuscripts at

http://www.hindawi.com
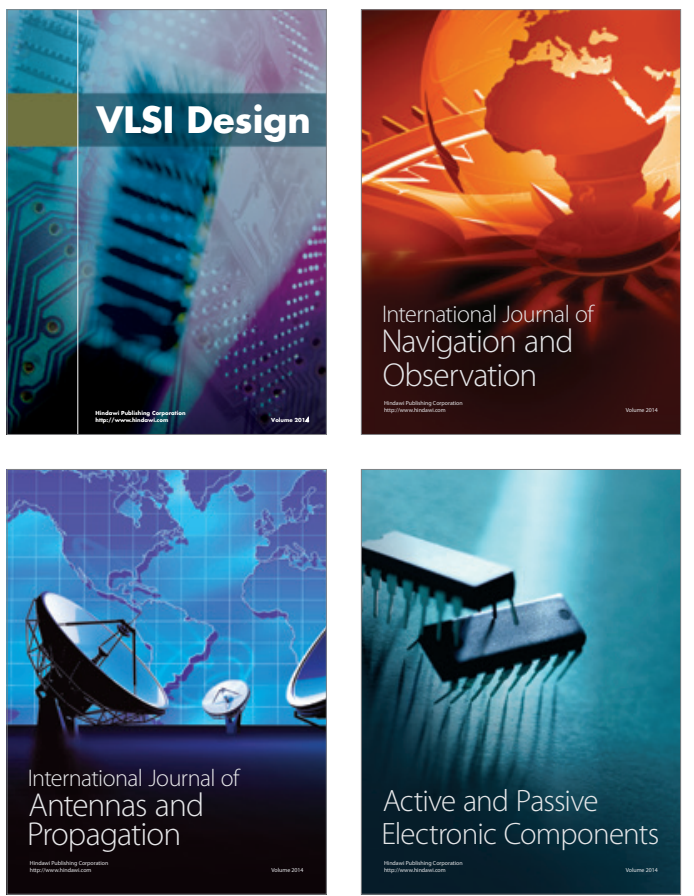
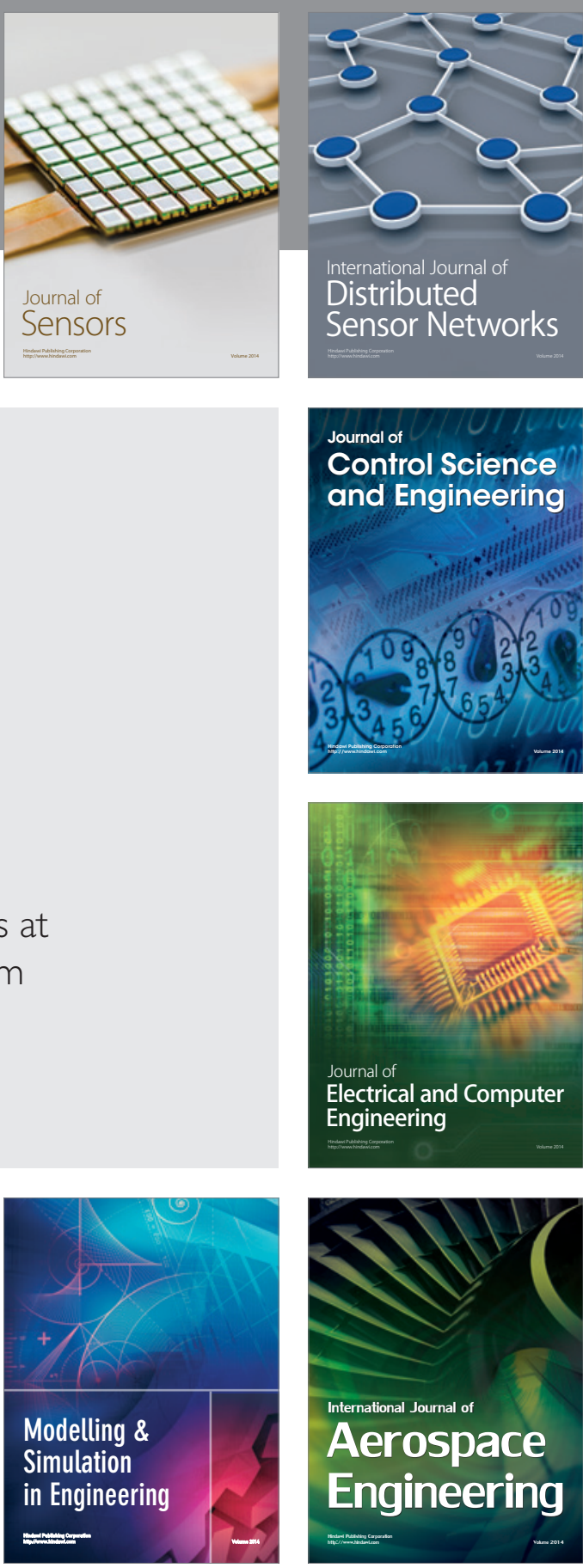

International Journal of

Distributed

Sensor Networks

Journal of

Control Science

and Engineering
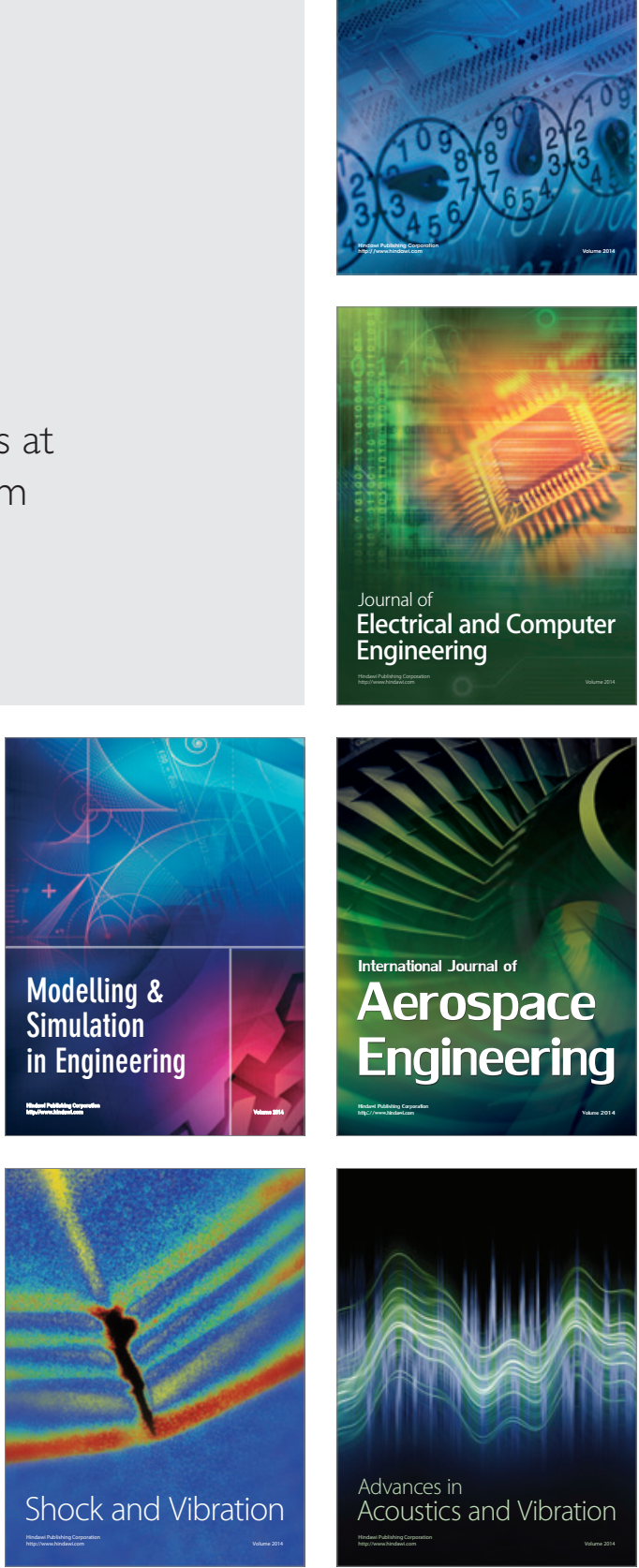